\title{
Dhima za Kipragmatiki za Kialami Pragmatiki 'ah' katika Mazungumzo ya Kiswahili
}

\author{
Magreth J. Kibiki ${ }^{1}$ \\ Chuo Kikuu Kishiriki cha Elimu cha Dar es Salaam (DUCE) \\ Pendo S. Malangwa ${ }^{2}$ \\ Chuo Kikuu cha Dar es Salaam.
}

\begin{abstract}
This study examines the pragmatic functions of the pragmatic markers ah in spoken Kiswahili. The data has been obtained from informal conversations made by Swahili speakers in informal social settings. These include 'vijiwe vya kahawa' (setting of informal conversations created around people drinking coffee) and 'vijiwe vya mamantilie' (setting of informal conversations around women preparing and selling food on the streets). Using Conversation Analysis (Sacks, 1962) and Contextualization Theory (Gumpers, 1982), the results show that the pragmatic marker ah conveys different meanings depending on context of use and hence has various pragmatic functions. Among the pragmatic functions identified in this article are; to be used as gap filler (the interlocutor is thinking about what to say), is used to start the conversation, is used to disagree with what the other interlocutor has said, to show that the speaker has changed from the state of not knowing to the state of knowing (Now I get you) and to show exclamation. Interestingly, also, the study shows that intonation and other paralinguistic features (like gestures) play a role in determining the pragmatic functions of this marker. Generally, this article concludes that pragmatic markers in spoken Kiswahili are rich in meanings. Therefore, they are wealthy to be investigated.
\end{abstract}

\section{Iksiri}

Makala haya yanakusudia kuchunguza kuhusu dhima za kipragmatiki za kialami pragmatiki (KIPRA) ah katika mazungumzo ya Kiswahili. Data za makala haya zimekusanywa kutoka katika mazungumzo yasiyo rasmi yanayofanywa na wazungumzaji wa Kiswahili katika maeneo yasiyo rasmi kama vile kwenye vijiwe vya kahawa na vijiwe vya mamantilie. Uchambuzi wa data za makala haya umeongozwa na Nadharia ya Usasanyuzi Mazungumzo ya Sacks ya mwaka 1962 na Nadharia ya Umuktadhaishaji ya Gumpers ya mwaka 1982. Matokeo ya uchunguzi huu yanaonesha kwamba KIPRA ah kina tabia ya kuchanuza maana tofautitofauti kulingana na

\footnotetext{
${ }^{1}$ Magrieth Kibiki is an assistant lecturer at Dar es Salaam University College of Education, A Constituent College of the University of Dar es Salaam. She holds MA and is currently a PhD student at University of Dar Es Salaam. She has specialized in Linguistics and she has authored several articles in semantics and Pragmatics. Her research Interests includes Semantics, Pragmatics and Conversation Analysis. E-mail: kibiki65@gmail.com
}

${ }^{2}$ Pendo Salu Malangwa is a senior lecturer and Director of Quality Assurance at the University of Dar es Salaam. She teaches in the Department of Kiswahili Language and Linguistics, Institute of Kiswahili Studies of the University of Dar es Salaam. She holds M.A.and PhD (Linguistics) from University of Dar es Salaam.Her areas of specialization and research interest include Translation, Interpreting, Terminology, Semantics and Pragmatics and has authored several papers in her areas of specialization. Email: pmalangwa@yahoo.co.uk. 
muktadha kinamotumika. Tabia hiyo hukifanya KIPRA hicho kuwa na dhima mbalimbali za kipragmatiki. Baadhi ya dhima za kipragmatiki tulizozibaini katika makala haya ni kama vile KIPRA ah kutumika kuashiria kuwa mzungumzaji anatafuta jambo la kusema, anaanzisha mazungumzo, anakataa au kukanusha jambo lililosemwa, ametoka katika hali ya kutofahamu au kutoelewa jambo kwenda kwenye hali ya kufahamu au kulielewa jambo hilo, na kuashiria kuwa mzungumzaji anashangaa. Aidha, makala haya yamebaini kwamba kiimbo na viashiria vingine vya kimuktadha kama vile ishara za viungo vya mwili vina mchango katika kubaini dhima za kipragmatiki za KIPRA ah. Kwa ujumla makala haya yanadokeza kwamba VIPRA vina dhima mbalimbali katika lugha ya Kiswahili na ni eneo ambalo linadokeza utajiri katika masuala ya utafiti wa kipragmatiki.

\section{Utangulizi}

VIPRA ni maneno au virai kutoka katika kategoria mbalimbali za kisintaksia, kama vile vielezi, viunganishi, vitenzi, vihisishi na virai vihusishi. Dhima ya maneno au virai hivyo ni kusaidia kumuelewesha mshiriki wa mazungumzo kufasiri uhusiano baina ya sentensi inayosemwa sasa na iliyosemwa kabla au baadaye katika kilongo cha mazungumzo. VIPRA huwa na maana zake za msingi ambazo ni za kimchakato ${ }^{3}$. Maana hizo hutajirishwa na muktadha vinamotumika. VIPRA havibadili maana ya msingi ya sentensi wala haviathiri masharti ukweli ya sentensi inayohusika. Huweza kujitokeza mwanzoni, mwishoni na hata katikati ya sentensi. Aidha, VIPRA huweza kuondolewa katika sentensi bila kuathiri maana ya msingi ya sentensi inayohusika (Nasir, 2017:15). Kupitia fasili ya Nasir (2017) ni wazi kuwa VIPRA ni vipashio muhimu kimawasiliano na vinatoka katika kategoria mbalimbali za kileksika. Miongoni mwa kategoria hizo ni vihisishi kama vile yeah (ndiyo/sawa), oh, okey (sawa), ah na no (hapana) (Norrick, 2009). VIPRA kutoka katika kategoria ya vihisishi vina upekee kisarufi (Cuenca, 2000). Upekee huo unadhihirika kifonolojia, kimofolojia, kisemantiki na kipragmatiki. Kifonolojia, VIPRA aina ya vihisishi hukiuka kaida za mfumo wa sauti wa lugha inayohusika. Hii ina maana kwamba VIPRA huwa na mfumo wake mahususi wa sauti tofauti na mfumo wa kawaida wa sauti za lugha mahususi. Hali hii huvifanya VIPRA hivyo kuwa na sifa za kiarudhi ambazo ni mahususi kwa VIPRA hivyo tu (Ameka, 1992; Cuza, 2015). Akitoa mfano katika lugha ya Kiingereza Ameka (1992) anafafanua zaidi kuwa baadhi ya VIPRA katika kategoria hii husemwa bila kuwa na irabu yoyote ( kwa mfano psst! na sh!). Aidha, huweza kusemwa kwa kuendeleza sauti (kwa mfano Ooooh). Kwa upande mwingine, Cuza (2015) anadokeza kuwa mabadiliko ya kiimbo kwenye VIPRA hivyo huashiria pia mabadiliko ya maana. Hii ina maana kwamba kiimbo kina athari kwenye maana zinazochanuzwa na VIPRA hivyo (Norrick, 2009). Kadiri VIPRA hivyo vinavyosemwa kwa namna tofautitofauti ndivyo maana zake zinavyobadilika.

Kimofolojia, VIPRA aina ya vihisishi vina sifa ya kutobeba viambishi awali wala tamati kama ilivyo kwa kategoria nyingine kama vile vitenzi na nomino (Ameka, 1992; Cuenca, 2000). Aidha, kisemantiki VIPRA vingi katika kategoria hii huwa havina maana za msingi au maana za kileksika. Hii ni kwa sababu VIPRA hivyo havina vijenzi sementiki bayana vinavyoweza kutumika kuvifafanua kama ilivyo kwa maneno kama meza, kiti au mama. Maana zake hutegemea nini kimesemwa kabla au baada ya KIPRA hicho katika kilongo cha mazungumzo (Norrick, 2009, Mykhaylenko, 2016).

Kisintaksia, VIPRA aina ya vihisihi hujitenga na vipashio vingine vya sentensi kwa kuwekea alama ya mkato (Norrick, 2009). Aidha, VIPRA hivyo huweza kufanya kazi kama neno na kama sentensi au kama tamko lenye maana ( Ameka, 1992; Cuenca, 2000). Hii ina maana kwamba VIPRA hivyo vina sifa ya kuwa neno (mfano vinapotumiwa ndani ya sentensi) au sentensi ( kwa

\footnotetext{
${ }^{3}$ VIPRA hurejelewa kuwa vina maana za kimchakato kwa sababu havina vijenzi semantiki bayana vinavyotumika kuvifafanua kama ilivyo katika maneno baba, meza na kadhalika. Maana zake za msingi hutegemea namna kipashio hicho kinavyosaidia kulifasiri tamko la sasa kwa kuhusianisha na tamko la awali au linalofuatia (Fraser, 1996).
} 
mfano kinaposimama peke yake kama tamko linalojitosheleza kimaana). Aidha, Ameka (1992) anasisitiza kuwa kitu pekee kinachotofautisha VIPRA aina ya vihisishi na kategoria nyingine kama vielezi na vihusishi ni ule uwezo wake wa kusimama peke yake kama tamko linalojitosheleza kimaana. Anasisitiza kuwa hata kama havina muundo wa kiima kiarifu (muundo unaochukuliwa kuwa ndiyo muundo msingi wa sentensi) bado vina sifa hiyo ya kuwa sentensi.

Kipragmatiki, VIPRA aina ya vihishishi vina sifa ya upolisemia (Matei, 2010). Dhana ya upolisemia kama ilivyotumiwa na Matei (2010) inahusu hasa ule uwezo wa VIPRA kuchanuza maana tofautitofauti kulingana na muktadha vinamotumika. Tabia hiyo huvifanya VIPRA kuwa na dhima mbalimbali za kipragmatiki (Ameka, 1992; Cuza, 2015; Matei, 2010). Kwa mfano, Ameka (1992) anafafanua kuwa VIPRA hivyo hutumika kuashiria hisia na mitazamo mbalimbali ya wazungumzaji. Kwa upande mwingine, Ameka (1992), Matei (2010) na Mykhaylenko (2016) wana mtazamo sawa kuwaVIPRA hivyo hutumika pia kuashiria hali ya akili ya mzungumzaji. Hii ina maana kwamba VIPRA hivyo hutumika kuashiria kama mzungumzaji ameelewa jambo linalozungumzwa au amekumbuka jambo fulani (Ameka, 1992). Aidha, Norrick, (2009) anaeleza dhima nyingine ya VIPRA hivyo kuwa hutumika kuanzisha mazungumzo na kujenga ushikamani wa mawazo katika mazungumzo.

Kwa ujumla, ufafanuzi unaotolewa na Ameka (1992), Cuenca (2000), Norrick, (2009) Matei (2010), Cuza, (2015) na Mykhaylenko (2016) umetusaidia kuelewa kuwa VIPRA kutoka katika kategoria ya vihisishi vina upekee katika kuchanuza maana. Hata hivyo, Ameka (1992), Cuenca, (2000) na Cuza, (2015) wote wanakubaliana kwamba namna VIPRA hivi vinavyochanuza maana ni tabia mahususi katika lugha mahususi. Hii ina maana kwamba kila lugha ina upekee wake katika namna VIPRA hivyo vinavyochanuza maana. Hivyo basi, ni wazi kuwa kuna haja ya kuvichunguza VIPRA hivyo katika lugha mahususi. Makala haya yanajikita katika kuchunguza dhima za kipragmatiki za KIPRA ah katika mazungumzo ya Kiswahili. KIPRA ah kimeteuliwa kama uchunguzi kifani ili kuweza kufafanua dhima hizo. Kwa hiyo, ni matarajio yetu kwamba matokeo ya utafiti huu yanaweza kutumika kuakisi dhima za kipragmatiki za VIPRA vingine katika lugha ya Kiswahili. Aidha, KIPRA hicho kimeteuliwa kwa sababu kinaonekana kuwa na tabia ya kuchanuza maana tofautitofauti kulingana na muktadha kinamotumika na hivyo kuwa na dhima mbalimbali za kipragmatiki.

\section{Mapitio ya Maandiko}

Katika mjadala wa mapitio ya maandiko, tutaangalia tafiti mbalimbali zilizofanywa kuhusu dhima za kipragmatiki za KIPRA ah katika lugha mbalimbali ulimwenguni pamoja na mjadala wa nadharia zinazohusu VIPRA na ambazo zimeongoza utafiti huu. Mjadala hasa utalenga katika kupambanua pengo au upungufu uliopo katika maandiko yaliyotangulia kwa lengo la kuonesha umuhimu wa kufanyika kwa utafiti huu. Aidha, mapitio yanatumika kuonesha uhusiano wa utafiti huu na tafiti zilizotangulia.

Tafiti kuhusu Dhima za Kipragmatiki za KIPRA ah katika Lugha mbalimbali Ulimwenguni KIPRA ah ni miongoni mwa VIPRA ambavyo havijapewa umuhimu kiutafiti. KIPRA hicho kinaonekana kutajwa na watafiti kama vile Ameka (1992) na Norrick (2007) kuwa kina sifa ya kuchanuza maana kulingana na muktadha kinamotumika. Hata hivyo, kwa kuwa lengo la wataalamu hawa lilikuwa ni kuvifafanua VIPRA aina ya vihisishi kwa ujumla, hatupati ufafanuzi wa kina kuhusu dhima za kipragmatiki za KIPRA hicho. Utafiti ambao unaonekana kukichunguza KIPRA ah kwa mwelekeo unaoendana na utafiti huu ni utafiti wa Choe (2015). Katika utafiti wake, Choe (2015) anajikita katika kuchunguza mbinu zinazotumiwa kuwakilisha kiimbo pindi KIPRA of (ah) kinapotumiwa kutuma ujumbe katika mitandao ya kijamii. Matokeo ya utafiti yanaonyesha kuwa wazungumzaji wanapoandika ujumbe wenye KIPRA of (ah) hutumia mbinu mbalimbali 
kuwasilisha kiimbo. Miongoni mwa mbinu anazozibainisha Choe (2015) ni kama vile kurefusha irabu na kuirudia sauti hiyo.

Utafiti huu umetusaidia kuelewa kwamba kiimbo kina athari kwenye maana zinazochanuzwa na KIPRA ah. Hata hivyo, utafiti wa Choe (2015) unahusu KIPRA ah katika lugha ya kikorea na si lugha ya Kiswahili. Aidha, utafiti wa Choe (2015) unahusu lugha ya maandishi na si lugha ya mazungumzo kama ilivyo katika utafiti huu. Vilevile, utafiti wa Choe (2015) haukujikita katika kuchunguza dhima za kipragmatiki za KIPRA of (ah) bali ulijikita katika kuchunguza namna kiimbo kinavyowasilishwa kiotograpindi KIPRA hicho kinavyotumiwa katika mitandao ya kijamii. Tofauti na utafiti wa Choe (2015), utafiti huu unajikita katika kuchunguza dhima za kipragmatiki za KIPRA ah katika mazungumzo ya Kiswahili.

Kwa upande wa lugha ya Kiswahili, kuna Kamusi mbalimbalimbali ambazo zimejaribu kubainisha maana za KIPRA ah. Miongoni mwa kamusi hizo ni kama vile BAKIZA (2010), TUKI (2012), BAKITA (2017) na VIDE MUWA (2018). Hata hivyo, ilibainika kuwa kuna changamoto katika ubainishaji huo. Changamoto inayojitokeza ni kwamba kamusi hizo zimechukulia kuwa namna tofauti za kusema KIPRA ah kama vile aa, a-a, ah, na ahaa kuwa ni VIPRA vinne tofauti. Data ya mazungumzo kutoka katika vijiwe vya kahawa na vya mamantilie inaonesha kwamba huo ni usemaji tofauti wa KIPRA hichohicho kimoja unaotokana na athari ya kiimbo kwenye KIPRA hicho. Aidha, KIPRA kinachochukuliwa kama ni KIPRA aa au a-a, hakisemwi kwa kutamka sauti /a/ katika uasili wake bali sauti /a/ hutamkwa ikiambatana na sauti /h/ yenye msikiko hafifu. Kama tulivyokwisha jadili katika utangulizi kuwa Kifonolojia, VIPRA aina ya vihisishi vinaonekana kukiuka kaida za mfumo wa sauti wa lugha inayohusika. Hivyo ni dhahiri kuwa tunapaswa kuzingatia kwamba VIPRA hivyo huwa na mfumo wake mahususi wa sauti ambao ni tofauti na mfumo wa kawaida wa sauti za lugha mahususi. Makala haya yanajenga hoja kuwa tofauti za kiotografia kama zinavyojitokeza katika kamusi ni kielelezo kuwa KIPRA hichohicho kimoja kinasemwa kwa namna tofautitofauti kutokana na athari za kiimbo. Hivyo basi, makala haya yatatumia KIPRA ah kama umbo msingi na tutafafanua katika uchambuzi wa data namna kiimbo kinavyoathiri dhima za kipragmatiki zinazobebwa na KIPRA hicho.

\section{Nadharia}

Utafiti huu umeongozwa na Nadharia mbili tofauti. Nadharia hizo ni Nadharia ya Usasanyuzi Mazungumzo na Nadharia ya Umuktadhaishaji. Mwasisi wa Nadharia ya Usasanyuzi Mazungumzo ni Harvey Sacks miaka ya 1960 (Sacks na wenzie, 1992). Madai makuu ya Nadharia ya Usasanyuzi Mazungumzo ni kuwa mazungumzo ni kitendo cha ushirika ambacho mzungumzaji na msikilizaji wanapewa umuhimu sawa katika kufanikisha maana ya kinachosemwa. Mzungumzaji hulipangilia tamko linalowasilishwa kwa msikilizaji na msikilizaji huliitikia tamko lililotolewa na mzungumzaji kwa kutoa mrejesho. Kwa mujibu wa nadharia hii, maana ya umbo lolote la kiisimu huafikiwa ndani ya mchakato wa mazungumzo yenyewe. Hii ina maana kwamba maana haitokani na umbo la kiisimu pekee, bali hutegemea namna washiriki wa mazungumzo wanavyolitumia umbo hilo la kiisimu katika mazungumzo. Nadharia hii inasisitiza pia kuwa maana ya neno si tuami bali huweza kurekebishwa na kuafikiwa na washiriki wa mazungumzo. Hivyo, maana hutegemea ushahidi unaopatikana ndani ya mazungumzo yenyewe kwa kuhusisha na nini kimesemwa kabla na baada ya hicho kinachosemwa sasa. Tumeteua nadharia hii kwa sababu maana za KIPRA ah zinaonekana kukitwa zaidi katika mazungumzo kwa kuzingatia nini kimesemwa kabla au baada ya kile kinachosemwa sasa. Hata hivyo nadharia hii haizungumzii sana kuhusu vipengele vingine vya kimuktadha ambavyo ni muhimu katika kuchanuza maana za KIPRA hicho. Hivyo basi, tutatumia pia nadharia ya umuktadhaishaji ya Gumperz (1982) ili kuziba pengo hili. 
Nadharia ya Umuktadhaishaji ni nadharia iliyoasisiwa na Gumpers mwaka 1982. Nadharia hii inasisitiza zaidi kuzingatia viashiria mbalimbali vya kimuktadha katika kung'amua maana ya kinachosemwa. Kwa mujibu wa Gumperz (1982), viashiria mbalimbali vya kimuktadha ni malighafi muhimu kwani husaidia katika kung'amua maana iliyokusudiwa na mzungumzaji. Maoni ya Gumpers (1982) ni kwamba viashiria hivyo vya kimuktadha vinajumuisha mada inayozungumziwa, hali, dhima za wazungumzaji, mahusiano yao, uhusiano baina ya mzungumzaji na mada inayozungumwa, ujumbe unaowasilishwa, matendo yanayoambatana na usemi kama vile ishara za viungo vya mwili na namna tamko linavyosemwa kwa kutumia viarudhi kama vile kiimbo, kidatu, mkazo na wakaa. Hivyo basi maana ya neno hutegemea muktadha mzima ambamo neno hilo limetumika. Nadharia hii imeteuliwa kwa sababu vipengele mbalimbali vya kimuktadha vinavyosisitizwa katika nadharia hii kama vile ishara za viungo vya mwili na viarudhi vinaonekana vina mchango katika kubaini dhima za kipragmatiki za KIPRA ah.

Hivyo basi, Nadharia tete ya utafiti huu ni kwamba dhima za kipragmatiki za KIPRA ah zimekitwa zaidi katika muktadha wa mazungumzo. Hii ina maana kwamba maana za KIPRA hicho hutegemea nini kimesemwa na washiriki wa mazungumzo kabla au baada ya hicho kinachosemwa sasa, kimesemwa kwa namna gani, kimesemwa wakati wazungumzaji wakiwa katika hali gani, na je kuna ishara au matendo mbalimbali yanayoambatana na mazungumzo hayo?. Kwa hiyo, tutaona katika mjadala wa matokeo ya utafiti huu dhima mbalimbali za kipragmatiki za KIPRA ah zikibainishwa kwa kuzingatia ushahidi unaopatikana ndani ya mazungumzo ya Kiswahili na katika muktadha halisi.

\section{Mbinu}

Data za utafiti huu zimekusanywa kutoka katika mazungumzo yasiyo rasmi yanayofanywa na wazungumzaji wa lugha ya Kiswahili katika vijiwe vya kahawa na vya mamantilie. Vijiwe hivi viliteuliwa kwa sababu unywaji wa kahawa na shughuli za mamantilie huambatana na mazungumzo na hivyo, VIPRA hutumika zaidi. Eneo la utafiti lililotumika kupata vijiwe vya utafiti huu ni mikoa ya Dar es Salaam na Tanga. Mkoa wa Dar es Salaam uliteuliwa kama kiwakilishi cha mikoa mingine yenye vijiwe vya kahawa na vya mamantilie. Aidha mkoa huo uliteuliwa kwa sababu ya urahisi wa kupata vijiwe vya kahawa na vya mamantilie ambavyo ni muhimu kwa utafiti huu. Katika mkoa huu, data zilikusanywa kutoka katika wilaya ya Ubungo, kata ya Manzese. Kata hiyo iliteuliwa kwa sababu aina zote mbili za vijiwe vilivyotumika kupata data vilipatikana kwa urahisi na hivyo kurahisisha mchakato mzima wa kupata data. Vilevile, mkoa wa Tanga uliteuliwa kwa sababu ndimo ulimo mji wa Tanga ambao ni miongoni mwa miji iliyopo katika upwa wa pwani ya Bahari ya Hindi katika Afrika Mashariki inayohusishwa na chimbuko la lugha ya Kiswahili (Massamba, 2002). Hivyo, wakazi wengi wanazungumza Kiswahili asilia. Katika mkoa wa Tanga, utafiti ulifanyika wilaya ya Tanga-Mjini, kata ya Ngamiani-Kati. Kata hiyo iliteuliwa kwa sababu vijiwe vya kahawa na vya mamantilie vilipatikana kwa wingi ikilinganishwa na maeneo mengine ya mji huo.

Jumla ya vijiwe 20 vilitumika kupata data ya utafiti huu. Idadi hii iliteuliwa kwa sababu ilimwezesha mtafiti kupata data toshelevu zilizokidhi malengo ya utafiti wake. Vijiwe 10 katika kata ya Ngamiani- Kati na vijiwe 10 katika kata ya Manzese vilitumika kupata data. Kwa kila kata, vijiwe vitano vilikuwa vya kahawa na vijiwe vitano vya mamantilie. Pia, kijiwe kimoja kiliweza kurudiwa zaidi ya mara moja ili kuweza kupata data za kutosha. Vijiwe 20 vilivyotumika vilipatikana kwa kutumia mbinu ya usampulishaji tajwa. Watafiti waliwatumia wenyeji wa maeneo yaliyohusika kubainisha vijiwe vya kahawa na vya mamantilie vinavyopatikana katika maeneo yaliyoteuliwa. Ili kupata data kutoka katika vijiwe hivyo, mbinu ya ushuhudiaji-ushiriki ilitumika. Ushuhudiaji-ushiriki ni mbinu ya kukusanya data ambayo humwezesha mtafiti kupata data kuhusu shughuli 
mbalimbali za jamii katika mazingira asilia kwa kushuhudia na kushiriki moja kwa moja katika shughuli hizo (Kawulich, 2006).

Mbinu ya ushuhudiaji-ushiriki iliteuliwa kwa sababu ilirahisisha shughuli nzima ya kurekodi mazungumzo kutoka katika vijiwe. Mbinu hii iliwafanya watafitiwa wasiwe na woga kuliko wangerekodiwa wakati ambapo watafiti si mshiriki wa mazungumzo. Kupitia mbinu hii, mazungumzo yaliyofanyika katika vijiwe vya kahawa na vya mamantilie yaliweza kurekodiwa katika kinasa sauti. Data hiyo imetusaidia kubaini mabadiliko ya maana ya KIPRA mh katika Mazungumzo ya Kiswahili

Aidha, Data zimechambuliwa kwa kufuata mkabala wa kitaamuli. Mkabala wa kitaamuli ni ule ambao data huwasilishwa kwa kutoa maelezo au ufafanuzi kuhusu vipengele mbalimbali muhimu katika utafiti. Enon (1998) anaeleza kuwa, uwasilishaji wa data kwa kutumia mkabala wa kitaamuli ni mchakato binafsi sana ambao hautumii kanuni nyingi za kitakwimu. Hivyo katika makala haya, ufafanuzi kuhusu dhima za kipragmatiki za KIRA ah zimefafanuliwa kwa kutoa maelezo na ufafanuzi wa kina.

Uchambuzi huo umefanyika kwa kufutata hatua zifuatazo: kwanza kabisa, watafiti waalisikiliza kwa umakini na kwa kurudia mara kwa mara mazungumzo yaliyorekodiwa katika kinasa sauti ili kuteua mazungumzo ambayo yana data anayoikusudia. Hatua hiyo ilifuatiwa na mchakato wa unukuzi wa mazungumzo hayo. Unukuzi wa mazungumzo umefanywa kwa kutumia mfumo wa unukuzi wa Jaffersonian (Jeffersonian Transcription System) kama unavyoelezwa na Psathas na Anderson (1993) na kama unavyoonekana katika kiambatisho cha makala haya. Mfumo huu umeteuliwa kwa sababu ni mfumo wa unukuzi unaosisitiza kuzingatia kila kipengele katika mazungumzo ili kuleta athari sawa kwa msomaji. Baada ya kufanya unukuzi, watafiti walianza kufafanua dhima mbalimbali za KIPRA ah kwa kutoa mifano.

\section{Dhima za Kipragmatiki za KIPRA ah katika Mazungumzo ya Kiswahili}

Kwa mujibu wa data zilizokusanywa kutoka katika mazungumzo yaliyofanywa katika vijiwe vya mamantilie na vijiwe vya kahawa, KIPRA ah kilionenakana kuwa na dhima za kipragmatiki kama ifuatavyo;

KIPRA ah kilionekana kuwa na dhima ya kipragmatiki ambayo ni kuashiria kuwa mzungumzaji amekumbuka jambo au anatoka katika hali ya kutojua au kuelewa jambo kwenda katika hali ya kujua au kuelewa jambo hilo. Mfano wa data kutoka katika mazungumzo ya kijiwe cha mamantilie inadhihirisha dhima hiyo:

\section{Data 1:}

1. (Huku akielekeza kidole kwa mpita njia) Umemuona yule?

2. B. (( anamwangalia mhusika anayepita)) Ni nani?

3. A. Si baba (anataja jina) yule.

4. B. Ah:: baba (anataja jina) kumbe!.

5. A. Sasa?

6. B. Sikumtambua hata. Mbona kachakaa vile?

7. A. Laana ya mke si mchezo. Mi huwa nasema laana ya mke mbaya:.. Kamwacha mwanamke wake mzuri, mchapakazi unategemea nini?

8. B. Hakuwa vile jamani! ((huku akionesha kusikitika) ... 
Katika muktadha huu wa mazungumzo, mzungumzaji B anaonekana kutomjua vizuri mhusika anayerejelewa (Taz. mstari wa 2). Mara baada ya mzungumzaji A kutaja jina la mhusika, Mzungumzaji B anatumia KIPRA ah kuashiria kuwa sasa amemkumbuka mhusika (Taz. mstari wa 4). Katika muktadha huu KIPRA ah kinasemwa katika namna ambayo sauti /h/ inasemwa kwa uendelezi kidogo (kiotografia kinaweza kulejelewa kama ahaa). Hii inaashiria kuwa kiimbo kina athari katika kuashiria dhima ya kipragmatiki ya KIPRA hicho. Hivyo basi, ni wazi kuwa KIPRA ah katika muktadha huu kina dhima ya kipragmatiki ambayo ni kuashiria kuwa mzungumzaji amekumbuka jambo au anatoka katika hali ya kutojua au kuelewa jambo kwenda katika hali ya kujua au kuelewa jambo hilo.

Aidha, ilibainika kwamba KIPRA ah kina dhima ya kipragmatiki ambayo ni kuashiria kuwa mzungumzaji anapuuza au anabeza kilichosemwa na mzungumzaji mwingine. Mfano wa data kutoka katika mazungumzo yaliyofanywa katika kijiwe cha kahawa inadhihirisha dhima hiyo:

\section{Data 2:}

9. A. ...Nani aliwatetea?

10. B. Si mbunge? Mimi nna sehemu ya kwenda kuwatetea?

11. A. Unamsikia huyu? Yaani siku zote unapiga kelele hapa hutetei hapo?

12. B. Sina sehemu ya kwenda kulitetea suala langu likaeleweka.

13. A. Wewe unatoa maoni yako kikatiba, sio lazima uende bungeni.

14. B. Alaa:: nitoe wapi?

15. A. Ina maana mimi nikitoa hapa maoni yenu nikaenda kumwambia mtu mwingine sijasaidia?

16. B. A:::h umesaidia wapi?

17. C. Nikichikua hapa maoni yako uliyoyaongea nikaenda kuyaandika twiter sijasaidia?

18. B. Umesaidia nini hapo?

19. A:::h msikilizeni huyu. Eti haki yake ya kikatiba, 'anaiundermine'.

20. B. Sasa wewe ulitoa maoni kwenye katiba mpya si ndiyo?

21. A. Eh.

22. B. Mpaka leo ipo wapi? Ina maana maoni yetu yamepotelea hewani.

23. C. E:h yamepotelea hewani.

24. A. A:::h sasa huyu naye, sasa katiba mpya imefuata nini?

25. B. Si ndiyo hayohayo maoni?

26. C.Ha ha

27. A. Bwana eh! hebu nipe kahawa msinivuruge kichwa changu...

Katika muktadha huu, wazungumzaji wanaonekana kutofautiana juu ya mada inayozungumzwa (haki ya kutoa maoni). Katika mstari wa 16 na 17 wazungumzaji wanaonekana kutumia KIPRA ah kupuuza jambo liliosemwa na mzungumzaji mwingine. (sawa na kusema huyu naye anaongea nini?). Katika muktadha huu, KIPRA ah kinasemwa katika namna ambayo ni tofauti na miktadha mingine tuliyokwisha kuibainisha (Taz. kwa mfano mstari wa 4). llibainika kuwa katika muktadha huu KIPRA ah kinasemwa kwa namna ambayo sauti /a/ ndiyo inasemwa kwa uendelezi kidogo na kisha kufuatiwa na sauti /h/ yenye msikiko hafitu (kiotografia kinaweza kulejelewa kama aaah). Hivyo kwa kuzingatia ushahidi wa kimuktadha, yaani nini kimesemwa kabla na baada ya KIPRA hicho na namna kilivyosemwa, ni dhahiri kuwa KIPRA ah kinatumika kubeba dhima ya kipragmatiki ambayo ni kuashiria kuwa mzungumzaji anapuuza au anabeza kilichosemwa na mzungumzaji mwingine.

Kwa upande mwingine, matokeo ya utafiti huu yameonesha kwamba KIPRA ah kina dhima ya kipragmatiki ambayo ni kuashiria kuwa mzungumzaji anastuka baada ya kukumbuka jambo 
alilosahau kulifanya. Data, mbalimbali kutoka katika mazungumzo ya vijiwe tofauti tofauti vya mamantilie inadhihirisha dhima hiyo:

\section{Data 3:}

28. A....Mi natoka

29. B. Haya

30. A. ((Anatembea hatua kadhaa)) Ah! Nipe mkoba wangu huo. Kidogo niusahau.

31. B. (( Anachukua mkoba na kumpa))

32. A. ((Anachukua na kuondoka))

\section{Data 4:}

33. A. Umeniletea korosho ulizosema?

34. B. Ah! Nimesahau kabisa.

35. A. Ndio zako wewe....

\section{Data 5:}

36. A. ((mteja anaonekana amesimama akisubiri kitu) nipe basi.

37. Nini?

38. Si chenji! (akionekana kushangaa kuulizwa swali hilo)

39. Ah! Nisamehe. Nilidhani nimeshakupa ((anachukua pesa na kumpa)

40. ((Anaondoka)).

Kwa kuzingatia namna KIPRA ah kilivyotumiwa katika miktadha tofauti ya mazungumzo, utafiti huu ulibaini kuwa KIPRA hicho kina dhima ya kipragmatiki ambayo ni kuashiria kuwa mzungumzaji anastuka baada ya kukumbuka jambo alilosahau kulifanya. Ushahidi wa kimuktadha ni kuwa katika miktadha hii tofauti, wazungumzaji wanaonekana kutumia KIPRA hicho mara baada ya kugundua kuwa walisahau kufanya jambo fulani. Aidha, mazungumzo yanayoambatana na usemaji wa KIPRA hicho (kama vile "kidogo nisahau" Taz. mstari wa 30, "nimesahau kabisa" Taz. mstari wa 32 na nilidhani nilishakupa Taz. mstari 37) na matendo mbalimbali kabla na baada ya kutumia KIPRA hicho (kama vile kumpa chenji mteja Taz. mstari wa 39) vinathibitisha zaidi juu ya dhima hiyo. Aidha ilibainika kwamba katika miktadha hii tofauti ya mazungumzo KIPRA ah kilisemwa katika namna ambayo sauti /a/ ndiyo inayosikika zaidi kuliko sauti /h/. Aidha, sauti /a/ inasemwa kwa kukatizwa bila kuwa na mwendelezo wowote wa sauti hiyo. Hali hii inaashiria kuwa usemaji tofauti wa KIPRA hicho unaashiria dhima tofauti zinazobebwa na KIPRA hicho. Hivyo basi, katika muktadha huu wa mazungumzo KIPRA ah kilionekana kutumika kuashiria kuwa mzungumzaji anastuka baada ya kukumbuka jambo alilosahau kulifanya.

Aidha, matokeo ya utafiti huu yameonesha kwamba KIPRA ah pia kilionekana kuwa na dhima ya kipragmatiki ambayo ni mshangao. Data kutoka katika mazungumzo ya kijiwe cha kahawa inadhihirisha dhima hiyo:

\section{Data 6:}

41. A. Unajua mi sijaelewa, we:.. .Ujue nimeziona picha lakini kichwani mwangu nikajiuliza watu wametengeneza au? Ni kweli makontena yaliyofunguliwa vitu vile vilikwemo?

42. B. E:h!

43. C. Huyu jamaa jipu=

44. B.=Jamaa jipu.

45. A. Ina maana shule zinataka kuwa na mashamba yake?

46. C.Mashamba ya kulima si ndiyo? ha ha ha 
47. B. E:h maana sasa yale matrekta ya nini?

48. ((B, C wanacheka kwa muda))

49. A. Sasa:, ngoja kwanza, 'document' zinakuwaje? Si zinakuwa na vitu vyote vilivyomo mle?

50. B. 'Document' kitu ga::nii?

51. A. Ah! (Akionekana kushangazwa na taarifa hizo) kwani pale hakuna skana jamani::? kuona kuna nini?

52. B. Skana ndiyo wametumia kuviona vile vitu. Wakaamua kufungua kujiridhisha je ni kweli tunachokiona? Kufungua ndiyo wanakutana na matrekta.

53. A. Ah! hii kali...

Katika muktadha huu wa mazungumzo, mzungumzaji A anaonekana kuwa na shauku ya kutaka kujua ukweli wa jambo linalozungumzwa Mara baada ya kuthibitishiwa na wazungumzaji wengine kuwa jambo hilo ni kweli, anatumia KIPRA ah kama ishara ya kushangaa (Taz. mstari 51 na mstari wa 53. Ingawa KIPRA ah kilionekana kusemwa katika namna inayoendana na ile inayoashiria kuwa mzungumzaji anastuka baada ya kukumbuka jambo alilosahau kulifanya (Taz. mstari wa 30, 34 na 39), katika muktadha huu dhima inayojidhihirisha ni tofauti na hiyo. Katika muktadha huu wa mazungumzo KIPRA ah kinadhima ya kipragmatiki ya mshangao. Hii ina maana kwamba mzungumzaji anashangazwa na taarifa anazozisikia. Hivyo, ni dhahiri kuwa KIPRA ah kinaweza kusemwa kwa namna ile ile lakini kikachanuza maana tofauti. Muktadha wa matumizi, kwa kuzingatia nini kimesemwa kabla na baada ya KIPRA hicho pamoja na ishara mbalimbali za viungo vya mwili (kama vile mwonekano wa kushangaa) ndivyo husaidia kubaini dhima hiyo iliyokusudiwa na mzungumzaji.

Halikadhalika, matokeo ya utafiti huu yanaonesha kwamba KIPRA ah kina dhima ya kipragmatiki ambayo ni kunukuu mazungumzo yaliyosemwa na mtu mwingine. Mfano wa data kutoka katika kijiwe cha mamantilie unathibitisha dhima hiyo:

\section{Data 7:}

54. A. Hivi ((anataja jina la mtu)) alikupa pesa zako?

55. B. Mwenzangu we:: kila nikienda ananipiga Kiswahili tu a:h mimi nilikuwa na shida, mtoto alikuwa anaumwa" ilimradi tu asinipe hela yangu.

56. A. Mh! kweli pesa imekuwa adimu siku hizi...

Katika muktadha huu wa mazungumzo, mzungumzaji B anaonekana kuanza kutumia KIPRA ah kabla ya kuanza kusema kilichosemwa na mzungumzaji mwingine (Taz. mstari wa 55). Hii inadhihirisha kuwa KIPRA ah kina dhima ya kipragmatiki ambayo ni kunukuu mazungumzo yaliyosemwa na mtu mwingine. Aidha, katika muktadha huu KIPRA ah kilionekana kusemwa kwa mwendelezo kidogo (kiotografia kinaweza kulejelewa kama aah) ikilinganishwa na kilivyosemwa awali katika mstari wa 16 na 24. Hii inadhihirisha kwamba mabadiliko ya kiimbo yana athari kwenye dhima za kipragmatiki zinazobebwa na KIPRA hicho.

Vilevile utafiti huu ulibaini dhima za kipragmatiki tofauti na tulizozibainisha hapo awali. Katika miktadha mingine ya mazungmzo, KIPRA ah kilionekana kutumika kuashiria kuwa mzungumzaji amekata tamaa. Data ya mazungumzo yaliyofanywa katika vijiwe vya mamantilie inathibitisha juu ya dhima hiyo:

\section{Data 8:}

57. A. Yaani leo hata senti kumi ya kula sina.

58. B. Hivi, kwa nini usianze kupika hata chapati tu. Zitakusaidia walau hela ya kula. Sio sawa na kukaa tu. 
59. A. Ah!, mtaji wenyewe uko wapi? Shida tupu...

\section{Data 9:}

60. A. Vipi ulienda vikoba?

61. B. Eh, nilienda.

62. A.Wamesemaje kuhusu zile hela?

63. B. Ah! Majibu yale yale tu.Tusubiri watatuita....

Katika data hizi, ni wazi kuwa KIPRA ah kimetumika kuashiria dhima ya kipragmatiki ambayo ni kukata tamaa. Muktadha wa matumizi, kwa kuzingatia nini kimesemwa kabla na baada ya KIPRA hicho vinathibitisha juu ya dhima hiyo. Kwa mfano maneno ya kukata tamaa kama vile "shida tupu "(Taz. mstari wa 59) na "majibu ni yale yale tu" (Taz. mstari wa 63) yanathibitisha zaidi juu ya dhima ya kipragmatiki ya KIPRA ah ambayo ni kukata tamaa.

Katika miktadha mingine tofauti ya mazungumzo, KIPRA ah kilionekana kuwa na dhima ya kipragmatiki ambayo ni kutumika kama kianzilishi cha mazungumzo. Data kutoka katika kijiwe cha kahawa inaashiria dhima hiyo:

\section{Data 10:}

64. A.... (( Anafika na kuwakuta washiriki wengine wakinywa kahawa) Ah::: wazima jamani?

65. B. [[Wazima]]

66. C. [[Sisi wazima]]

67. A. Asalam Aleykum.

68. B. [[Aleykum salaam]]

69. C. [[Asalam Aleykum]]...

Katika muktadha huu wa mazungumzo, KIPRA ah kilionekana kuwa na dhima ya kipragmatiki ambayo ni kuanzisha mazungumzo. Mzungumzaji A anaonekana kutumia KIPRA hicho mara anapofika na kabla hajasalimia washiriki wengine wa mazungumzo (Taz. mstari wa 64). Katika muktadha huu wa mazungumzo, KIPRA ah kinaonekana kusemwa katika namna ambayo sauti /h/ ndiyo inayosemwa kwa uendelezi zaidi tofauti na ilivyokuwa awali katika mstari 16 na mstari wa 24 (Kiotografia inaweza kulejelewa kama ahaaaa) Hivyo, ni wazi kuwa mabadiliko ya kiimbo yanasababisha pia dhima za kipragmatiki zinazobebwa na KIPRA hicho kubadilika. Aidha, mabadiliko hayo ya kiimbo yametusaidia kubaini dhima ya kipragmatiki iliyobebwa na KIPRA hicho.

Watafiti walibaini dhima nyingine ya kipragmatiki ya KIPRA ah ambayo nni kuashiria kuwa mzungumzaji amekasirika kutokana na kilichosemwa au kutendwa. Mfano wa data kutoka katika mazungumzo ya kijiwe cha mamantilie yanadhihirisha dhima hii:

\section{Data 11:}

70. A. Mama yupo?

71. B. Ametoka sasa hivi.

72. A. Si nimempigia simu sasa hivi kaniambia yupo hapa?

73. B.Katoka sio muda.

74. A. $\underline{\mathrm{AH}}$ !(akionekana kukasirika) mimi sasa ninachelewa. Ndio maana nilimpigia kabisa kabla sijaja. Watu wengine bwana hawajui kutunza muda kabisa::...

Data 12:

75. A. (Anafika kijiweni na kuanza kumtania mmoja wa mamantilie) Niambie mpenzi wangu.

76. B. Mpenzi wako nani? 
77. A. Si wewe? Hunitaki siku hizi eh! (Anamsogelea na kumshika mabegani)

78. B. (Akionekana kukasirika, anamtoa mikono kwa nguvu mabegani) $\underline{\mathrm{AH}}$ ! niache bwana!. Malaya mkubwa we:....

Katika miktadha hii ya mazungumzo, KIPRA ah kimeonekana kubeba dhima ya kipragmatiki ambayo ni kuashiria kuwa mzungumzaji amekarishwa na jambo fulani. Mazungumzo kabla na baada ya KIPRA hicho pamoja na ishara za mwili kama vile mwonekano wa kukasirika pamoja na namna KIPRA hicho kilivyosemwa (kwa kuwekwa mkazo na kuongeza sauti Taz. mstari wa 74 na 78) vilitusaidia kubaini kuwa KIPRA ah kina dhima hiyo ya kuashiria kuwa mzungumzaji amekarishwa na jambo fulani.

Vilevile, kupitia uchunguzi wa vijiwe vya kahawa, ilionekana kwamba dhima nyingine ya kipragmatiki ya KIPRA ah ni kutumika kuashiria kuwa mzungumzaji anatafakari, anajaribu kukumbuka jambo au anatafuta cha kusema. Data kutoka katika kijiwe cha kahawa inadhihirisha dhima hii:

\section{Data 13:}

79. A. ...Ngoja kwanza, sasa sisi tunapofuturu na Tunduma wanafuturu muda huohuo?=

80. B. Ah ah=

81. C.= Kwa nini?. Kwa nini na simu tunazo kwanini tusiwapigie simu haloo sisi huku tunafuturu na nyinyi huko futulini huko=

82. D. Na Tanzania ni moja hii=

83. B. Swali hili ungemuuliza huyu (anamwonyesha mzungumzaji A)

84. A. Jamani mimi nimejenga hoja gani::? Mimi nimetoa mtazamo wa watu waliokuwa wanahoji hayo.

85. B. Hao si watu tu

86. A.Na wewe si mtu tu, [[ah ah na wewe si mtu tu]] kwani wewe malaika?

87. [[we umesikia]]

88. D. Sikiliza kitu kimoja, kwani nabii lbrahim alikuwa dini gani?

89. A. Muislam

90. D. Yesu kristo ilikujaje?

91. A. Imekujaje?

92. D. Eh

93. A::h (0.5) wale a::h (0.7) ni jinsi walivyopokea mapokeo yao. Wamepokea dini wakaweka na mambo yao ndiyo hivyo unavyoiona. Ndiyo maana sisi na marafiki zetu hawa wa damu tunapishana sehemu ndogo tu. Hawa wanaposema mwana wa Mungu sisi tunasema hapana mtume wa Mungu. Ndiyo tunapopishana hapo. Wala hatuna tofauti kubwa...

Katika muktadha huu wa mazungumzo, wazungumzaji wanaonekana kujadiliana masuala mbalimbali yanayohusu dini. Katika mstari wa 12 mzungumzaji D anamuuliza swali mzungumzaji A na mzungumzaji A anajibu swali hilo huku akitumia KIPRA ah kuashiria kuwa anaonekana kuanza kujibu huku akitumia KIPRA ah kuashiria kuwa anatafakari au anatafuta jambo la kusema. Katika muktadha huu KIPRA ah kinasemwa taratibu huku kukifuatiwa na ukimya kidogo kuashiria kuwa mzungumzaji anatafakri au anatafuta jambo la kusema. Aidha, sauti /a/ inasemwa kwa mwendelezo kidogo na kisha kufuatiwa na sauti /h/ yenye msikiko hafifu. Hivyo ni dhahiri kuwa katika muktadha huu wa mazungumzo KIPRA ah kina dhima ya kipragmatiki ambayo ni kuashiria kuwa mzungumzaji anatafakari, anajaribu kukumbuka jambo au anatafuta cha kusema. 
Aidha, data iliyopatikana kutoka katika vijiwe vya mamantilie inaonesha kwamba KIPRA ah kina dhima nyingine ya kipragmatiki ambayo ni kukataza jambo fulani lisifanyike. Mifano ya data zifuatazo inadokeza juu ya dhima hiyo:

\section{Data 14:}

94. A. ...((mtoto anaonekana kuchezea kisu)).

95. B. ((mama anamwona mtoto akichezea kisu)) ah ah utajikata ((anamnyang'anya mtoto kisu na kuendelea na kazi))

96. A. ((mtoto analia))...

\section{Data 15:}

97. A. ...(Anaita kwa kutaja jina)

98. B. Abee.

99. A. Ongeza maji kwenye nyama.

100.B. ((Anachukua maji kwenye jagi na kuanza kumimina kwenye sufuria yenye nyama))

101.A. Ah ah usitie yote. Kidogo tu. Imeshaanza kuiva hiyo. Yanatosha.

102.B. ((Anaacha kuendelea kumimina maji) ...

Katika kilongo hiki cha mazungumzo, ingawa KIPRA ah kinaonekana kusemwa kwa uradidi kama ilivyosemwa katika data namba 11, ilibainika kwamba kuna tofauti ya dhima inayobebwa na KIPRA hicho. Katika muktadha huu, KIPRA ah kilionekana kutumika kuashiria kuwa mzungumzaji anakataza tendo fulani lisifanyike. Katika mstari wa 95 mzungumzaji B anatumia KIPRA ah kumkataza mzungumzaji A asichezee kisu. Aidha, katika mstari wa 101 data ya 13, mzungumzaji A anatumia KIPRA ah kumkataza mzungumzaji B asitie maji yote kwenye mboga. Matendo yaliyoambatana na mazungumzo hayo (kama vile kunyang'anya kisu na kuacha kumimina maji) pamoja na mazungumzo yanayofuatana na usemaji wa KIPRA hicho ni viashiria vya kumuktadha vilivyotusaidia kubaini dhima hiyo. Hivyo ni wazi kuwa KIPRA ah kina dhima ya kipragmatiki ambayo ni kukataza jambo fulani lisifanyike.

Katika miktadha mingine tofauti ya mazungumzo KIPRA ah kilionekana kubeba dhima ya kipragmatiki ambayo ni kuashiria utani au mzaha. Data ifuatayo yenye mazungumzo kutoka katika kijiwe cha kahawa inathibitisha juu ya dhima hiyo:

Data 16:

103.A .... Mini nasemea hivi:: yeye kwenda pale:: kwa sababu unapokwenda kwa mwenzio maana yake na wewe si una tabia ya kuita watu? Kwenye jambo lako lolote zuri na yeye si unamwita:

104. B. [[Na yeye akituma mwakilishi? ]]

105. C. [[Na yeye akituma mwakilishi? ]]

106. A. Anaona Sawa (0.5), we umeona tangu ameingia madarakani yeye amemwita mtu? Unajua yule, yule kama walivyosema watu diplomasia yule ni 'zero::'.

107. (Wanacheka kwa pamoja)

108.C. Shida nini hakuandaliwa au?

109. A. Hakuandaliwa ndiyo.

110. C. A:::h Fred bwana: ha ha ha

111.A. Haki ya Mungu tena......We ukiona kina Mkapa walikuwa wanapewa dakika kumi na tano umoja wa mataifa, yaani hata watu wakiongea vitu vinaingia akilini. Hupewi hivihivi. Mwingine alipewa tano, sijui raisi wa Zambia alishawahi kupewa dakika tatu akazira kuongea. Sasa ile muda anajishika koti sijui nini wakamwambia shuka.

112. A, B, C (( wanacheka kwa muda)

113. B. A:::h unaanza kujipapasa? Koti hukuliweka vizuri toka ulikotoka? Ha ha ha

114.C. Sisi hatujaja kukuangalia hapa. 
115.A. Tumekuja tusikilize maneno yako, una nini kipya? Unahitaji msaada gani?...

Katika muktadha huu wa mazungumzo, wazungumzaji wanaoanekana wakijadiliana kuhusu siasa. Katika mazungumzo hayo KIPRA ah kinaonekana kutumika kuashiria utani au mzaha (Taz. Mstari wa 110 na mstari wa 113. Kuwapo kwa vicheko vya mara kwa mara ni kiashirio kimojawapo cha kimuktadha kilichotusaidia kubaini dhima hiyo. Ingawa KIPRA ah kinaonekana kusemwa katika namna inayofanana na kilipotumiwa katika mstari wa 14 na 26 (data namba 2) kilipotumika kubeba dhima ya kuashiria kuwa mzungumzaji anapuuza au anabeza kilichosemwa na mzungumzaji mwingine, katika muktadha huu dhima tofauti ya kipragmatiki inajitokeza. Hali hii inaashiria kuwa KIPRA ah kinaweza kusemwa katika namna ile ile lakini kikachanuza maana tofauti kulingana na muktadha kilimotumika.

Vilevile, tuliweza kubaini dhima nyingine ya kipragmatiki ya KIPRA ah ambayo ni kutumika kuashiria kuwa jambo linalolejelewa halitatokea au haliwezi kutokea au ni vigumu kutokea. Data inayohusu mazungumzo kutoka katika kijiwe cha kahawa inathibitisha dhima hiyo:

\section{Data 17:}

116....A.Mimi nilishawahi kukataa kuhojiwa pale.

117.B. Unakataa? unakataa kuhojiwa?

118. C. Unaweza kukataa kuhojiwa.

119.D. A:h wapi, utahojiwa tu utake usitake.

120.B. Sikiliza, kuhojiwa unaweza ukakataa, ila polisi wana njia hizi mbili za kuhoji sawa?

121. ((Wanacheka kwa muda))

122. C.We mjinga wewe ha ha ha.

123. B. Wananjia halali sawa? Ya maelewano halafu wana ile ya kuforce utake usitake utahojiwa. Sasa lazima wewe- utakubali uumie ndiyo uhojiwe?

124.A. Ah wapi.

125. B. Eh ndiyo maana wengine wanaenda kupingia wapi mahakamani. Kwa sababu hata mahakama wanajua kwamba polisi ni police force hakuna kitu kinachofanyika polisi kwa uhalali. Wanatumia kufo kuforce. Ndiyo maana hata mahakama wakifika vilevilehaiwezekani mimi nikiri kufungwa miaka thelathini umenipa kitu gani wewe mimi nikiri. Nikiri-

126. (( wanacheka kwa muda)...

Katika muktadha huu wa mazungumzo, wazungumzaji wanaonekana kutofautiana juu ya uwezekano wa mtuhumiwa kukataa kuhojiwa. Kwa mfano, Katika mstari wa 118 mzungumzaji C anauliza "unaweza kukataa kuhojiwa?" na mzungumzaji D anajibu huku akitumia KIPRA ah sambamba na neno wapi kuashiria kuwa jambo hilo haliwezekani (Taz. mstari wa 119). Hivyo basi, katika muktadha huu wa mazungumzo, KIPRA ah kilionekana kuwa na dhima ya kipragmatiki ambayo ni kuashiria kuwa jambo linalozungumzwa haliwezekani au ni vigumu kutokea.

Katika miktadha mingine tofauti ya mazungumzo, KIPRA ah kilionekana kutumika kuashiria dhima ya kipragmatiki ambayo ni jibu la swali linaloashiria kukataa au kukanusha jambo. Dhima hiyo inajitokeza katika data ifuatayo kutoka katika mazungumzo yaliyofanyika katika kijiwe cha kahawa:

Data 18:

127.A. ...((Huku akimtazama mzungumzaji B))We umeoa?

128. B. Ah ah (anajibu huku akitikisa kichwa)). 
129. A. Kama hujaoa huwezi jua. Sasa, sisi wanaume tuliooa mara nyingine tunafanya mambo ya ngono nje ya ndoa nini, sasa yule mwanamke wa nje akikwambia huniridhishi, hata haikuumi.

130.B na C ((wanacheka kwa muda))

131.B. haikuumi?

132. A. Eh hainiumi, lakini ndio upo unakula chakula pale, watoto washalala, mkeo anakwambia lakini mme wangu::: ukinifanya mimi siridhiki, sitosheki. Mh::lazima ucheke ((anaigiza kicheko)). Lazima ujifarague kwamba unajua mke wangu: ninakuwa nimechoka na nini. unajibalaguza sasa hapo. Nakwambia hata ukilala husinzi:::.

133. A, B na C ((wanacheka kwa muda)).

134. C. Hulali siku hiyo.

135. A. Hulali, unajiulizaa mambo mengi, kama simridhishi ina maana atatafuta mtu mwingine amridhishe? Kwa hiyo, inabidi ujitahidi kwa mbinu zozote zile umridhishe.

136. B. Ushinde.

137. A. EH, kwa sisi tuliooa lazima mwanamke apate chakula kile: kitamu cha kwake. Kwa sababu ndiyo unaoekana wewe lijali na umempa mzigo wake...

Katika data hiyo, mzungumzaji $A$ anamuuliza mzungumzaji $B$ kama ameoa na mzungumzaji $B$ anajibu swali hilo kwa kutumia KIPRA ah akiashiria kuwa hajaoa (sawa na kusema hapana sijaoa). Katika muktadha huu KIPRA hicho kinasemwa kwa uradidi (mzungumzaji anakirudia mara mbili) kuashiria kuwa anakataa jambo. Dhima hiyo inathibitika zaidi pale mzungumzaji $A$ anapotikisa kichwa kama ishara ya kukataa. Aidha, kitendo cha mzungumzaji A kusema kuwa "wewe hujaoa kwa hiyo huwezi jua" (Taz. mstari wa 3) kunathibitisha zaidi kuwa KIPRA ah kimetumika kuashiria dhima hiyo. Hivyo ni wazi kuwa katika muktadha huu, KIPRA ah kilionekena kuwa na dhima ya kipragmatiki ambayo ni jibu la swali linaloashiria kukataa jambo.

llibainika pia kwamba, dhima nyingine ya kipragmatiki inayobebwa na KIPRA ah ni kutumika kumsaidia mzungumzaji kujenga hoja inayoashiria kupinga jambo lililosemwa na mzungumzaji mwingine. Hapa mzungumzaji anatumia KIPRA ah kuashiria kuwa jambo lililosemwa na mzungumzaji mwingine si sahihi au hakubaliani nalo na hivyo analikanusha au kulipinga. Mfano wa data kutoka katika mazungumzo ya vijiwe vya kahawa inadhihirisha dhima hii.

Data 19:

138. A. Wakati mwingine anaongea upuuzi lakini watu wanashangilia tu.

139. B. Watu wanashangilia tu.

140. C. Ah ah kushangilia watu wachache: kusifanye ikawa furaha ya watu wengi.

141. B. Ah ah kwani hata utafiti si unachukua sample?

142.C. Walioshangilia si watu wachache tu kwani ni nchi nzima. Kwani alipoenda bagamoyo akawaambia mashamba ya magereza warudishieni wananchi si watu walifurahia kule?

143.A. Limetekelezeka?=

144.C. =Lakini lile si kwa wachache tu. Kwani walifurahia nchi nzima?=

145. A. =Limetekelezeka?=

146. C. Walilolifurahia watu nchi nzima ambalo limeleta gumzo na kero kiserikali, wamachinga waacheni wafanye biashara maeneo [yo:te.]

147. B. [Wasigongwe tu na magari]=

148.C. $=$ Wasigongwe tu na magari. Lile ndio lilifurahiwa na watu wote.

149.A. (0.3) Ah ah mimi siwezi kukukatalia, si lina maslahi na watu wa chini?

150. C. Nakwambia lile ndio limefurahiwa na watu wote.

151. B. Ah ah baki hukohuko kwenye wengi [[ sio wote.]]

152. A.

[[Wenye maduka hawakufurahi lakini.]] 
153.B. Na lile wenye maduka mbona wamekasirika?Ukiona wanashangilia jiulize ni wenye nacho au wasio nacho. Mbona aliongea na majaji ,uliona wanashangilia? We vipi bwana?...

Katika muktadha huu, wazungumzaji wanaonekana kutumia KIPRA ah mara kadhaa (Taz. mstari wa ) kuashiria dhima ya kipragmatiki ambayo ni kumsaidia mzungumzaji kujenga hoja inayoashiria kupinga jambo lililosemwa na mzungumzaji mwingine. Dhima hiyo inaonekana kujitokeza zaidi katika muktadha ambao wazungumzaji wanatofautiana katika mitazamo juu ya jambo fulani. Katika muktadha huu wazungumzaji wanaonekana kutofautiana juu ya suala la wingi wa watu katika mikutano. Kwa hiyo, KIPRA ah kinatumika kujenga hoja huku kikitumiwa kuashiria kuwa mzungumzaji anapinga, anatofautiana au anakanusha kilichosemwa na mzungumzaji mwingine. Ushahidi wa kimuktadha ni kuwa mara baada ya kutumia KIPRA hicho mzungumzaji husika anafafanua kile anachodhani kuwa ni sahihi tofauti na kilichosemwa awali na mzungumzaji mwingine. Hivyo ni dhahiri kuwa KIPRA ah kimetumika kubeba dhima hiyo ya kumsaidia mzungumzaji kujenga hoja inayoashiria kukanusha au kupinga jambo lililosemwa na mzungumzaji mwingine.

\section{Hitimisho}

Kutokana na data yetu pamoja na uchambuzi tuliofanya hapo juu, ni wazi kuwa KIPRA eh kina dhima mbalimbali za kipragmatiki. Dhima tulizozipata kutokana na utafiti huu ni; KIPRA ah kutumika kuashiria kuwa mzungumzaji amekumbuka jambo au anatoka katika hali ya kutojua au kuelewa jambo kwenda katika hali ya kujua au kuelewa jambo hilo, kuashiria kuwa mzungumzaji anapuuza au anabeza kilichosemwa na mzungumzaji mwingine, kuashiria kuwa mzungumzaji anastuka baada ya kukumbuka jambo alilosahau kulifanya, kuashiria kuwa mzungumzaji anashangaa, kunukuu mazungumzo yaliyosemwa na mtu mwingine, kutumika kuashiria kuwa mzungumzaji amekata tamaa, kutumika kama kianzilishi cha mazungumzo, kuashiria kuwa mzungumzaji amekasirika au ameghafirika kutokana na kilichosemwa au kutendwa, kuashiria kuwa mzungumzaji anatafakari, anajaribu kukumbuka jambo au anatafuta cha kusema, kutumika kumsaidia mzungumzaji kujenga hoja inayoashiria kukanusha au kupinga jambo lililosemwa na mzungumzaji mwingine, kuashiria utani au mzaha, kuashiria kuwa jambo linalolejelewa halitatokea au haliwezi kutokea au ni vigumu kutokea, kutumika kama jibu la swali linaloashiria kukataa au kukanusha jambo na kutumika kumsaidia mzungumzaji kujenga hoja inayoashiria kupinga jambo lililosemwa na mzungumzaji mwingine Hata hivyo, ni vyema tukaweka bayana kuwa dhima tulizozijadili hapa ni kwa mujibu wa vyanzo viwili tu vya data; ambavyo ni vijiwe vya kahawa na vijiwe vya mamantilie. Upo uwezekano wa KIPRA ah kuwa na dhima nyingine zaidi ya tulizozijadili katika makala haya kama uchunguzi wa kina zaidi utafanyika.

Pia, makala haya yamebaini kwamba kiimbo kina mchango mkubwa katika kubaini dhima za kipragmatiki za KIPRA ah. Hii ina maana kwamba usemaji au utamkaji tofauti wa KIPRA ah unaonekana unachanuza dhima iliyokusudiwa na mzungumzaji. Hivyo basi, sifa za kiarudhi hususani kiimbo, zinaweza kuchunguzwa zaidi katika utafiti wa dhima za VIPRA. Halikadhalika, makala haya yamebaini kwamba viziada lugha kama vile ishara za viungo vya mwili vina mchango katika kubaini dhima inayobebwa na KIPRA ah. Hii ina maana kwamba usemaji wa KIPRA ah huambatana na ishara mbalimbali za viungo vya mwili ambazo ni muhimu katika kubaini dhima za kipragmatiki za KIPRA hicho. Kwa ujumla matokeo ya utafiti huu yanadokeza kwamba VIPRA vina dhima mbalimbali katika lugha ya Kiswahili na ni eneo ambalo linadokeza utajiri katika masuala ya utafiti wa kipragmatiki.

\section{Marejeleo}

Ameka, F. (1992). Interjections: The universal yet neglected part of speech. Journal of Pragmatics 8, 101-118. 
BAKITA. (2017). Kamusi kuu ya Kiswahili. Nairobi: Longhorn Publishers Limited. Toleo la 2.

BAKIZA. (2010). Kamusi la Kiswahili fasaha. Nairobi: Oxford University Press.

Choe, H. (2015). Ah...Ah! An exploration of the korean discourse marker 아 (ah) in instant messages (IM). Katika Buckeye East African Linguistics. 1-4.

Cuenca, M.J. (2000). Defining the indefinable? Interjections. Syntaxis, 3, 29-44.

Cuza, A. I. (2015). Language functions in a comparative classification of interjection. Potential benefits to their translation. Journal of Romanian Literary Studies, 6, 920-930.

Enon, J. C. (1998). Education research, statistics and measurements. Kampala: Makerere University.

Gumperz, J. (1982). Discourse strategies. Cambridge: Cambridge University Press.

Kawulich, B. B. (2006). Participant observation as a data collection method. Qualitative Social Research. Imesomwa tar. 25 Julai, 2018 kutoka katika http://www.qualitativeresearch.net/fgs/.

Matei, M. (2010). Discourse markers as functional elements. Philology and Cultural Studies, 3(52), 119-126.

Mykhaylenko. V. V. (2016). Interjection impact on discourse pragmatics. Series "Philology" Linguistics, 2(5), 28-31.

Nasir, S, H. (2017). The interpretation of discourse markers ya 'yes' and nggak 'no' in Indonesia". Tasnifu ya Umahiri (haijachapishwa). Chuo Kikuu cha Radboud.

Norrick, N.R. (2009). Interjections as pragmatic markers. Journal of Pragmatics, 41, 866-891.

Psathas, G., \& T. Anderson. (1993). The practices of transicription in conversation analysis. Semiotica, 78(1/2), 75-99.

Sacks, H., E. A., Schegloff \& Jefferson, G. (1992). Lectures on conversation. Oxford: Basil Blackwell.

VIDE MUWA, (2018). Kamusi Pevu ya Kiswahili. Nairobi: Vide Muwa Publishers Limited. Toleo la 2. 


\section{Kiambatisho}

Katika makala haya tumetumia alama mbalimbali zilizopendekezwa na Psathas na Anderson (1993) kama ifuatavyo:

\begin{tabular}{|c|c|}
\hline ALAMA & MAANA \\
\hline Namba 1- 101 & $\begin{array}{l}\text { Tamko la mzungumzaji mmoja au zamu } \\
\text { tofauti za kuzungumza. }\end{array}$ \\
\hline$A, B, C$ & Kuwakilisha Majina ya wazungumzaji. \\
\hline $\begin{array}{lrr}\text { Herufi kubwa } & \text { katika } \\
\text { silabi au } & \text { neno } \\
\text { (Mfano, EH } & \\
\end{array}$ & $\begin{array}{l}\text { Maneno yaliyosemwa kwa kuongezeka kwa } \\
\text { sauti kuashiria msisistizo. }\end{array}$ \\
\hline$((\quad))$ & $\begin{array}{l}\text { Kufafanua matendo yanayofanywa na } \\
\text { washiriki wa mazungumzo. }\end{array}$ \\
\hline$=$ & $\begin{array}{l}\text { Mfululizo wa uzungumzaji; mzungumzaji wa } \\
\text { pili ameanza kuzungumza mara tu baada ya } \\
\text { mzungumzaji wa kwanza kumaliza au } \\
\text { mzungumzaji huyohuyo ameongea jambo } \\
\text { moja na kisha akaongea jambo jingine bila } \\
\text { kupumzika. }\end{array}$ \\
\hline $\begin{array}{l}\text { Namba zilizowekwa } \\
\text { katika mabano kwa } \\
\text { mfano }(0.5) \text {. }\end{array}$ & Muda wa ukimya. \\
\hline$\left[\left[\begin{array}{ll}{[} & ]\end{array}\right]\right.$ & $\begin{array}{l}\text { Mazungumzo yanayotokea kwa wakati } \\
\text { mmoja (wazungumzaji wawili au zaidi } \\
\text { wanaanza kuzungumza kwa wakati mmoja. }\end{array}$ \\
\hline & $\begin{array}{lrr}\text { Mwingiliano } & \text { katika } & \text { mazungumzo } \\
\text { (mzungumzaji } & \text { mmoja } & \text { anaingilia } \\
\text { mazungumzo ya } & \text { mzungumzaji } & \text { mwingine. }\end{array}$ \\
\hline : & $\begin{array}{l}\text { Kiwango cha urefushaji wa silab. Semi } \\
\text { koloni zitaongezeka au kupungua } \\
\text { kutegemea na kiwango cha urefushaji wa } \\
\text { silabi husika. }\end{array}$ \\
\hline - & $\begin{array}{l}\text { Mstari unaowekwa chini ya neno au silabi } \\
\text { umetumika kuashiria mkazo (mfano njoo } \\
\text { hapa). }\end{array}$ \\
\hline$\underline{0}$ & $\begin{array}{l}\text { Imetumika kuashiria sauti inayotamwa kwa } \\
\text { kubembeleza au kwa upole. }\end{array}$ \\
\hline & Kiimbo cha kuendelea. \\
\hline$?$ & Kiimbo cha swali. \\
\hline ! & Kiimbo cha mshangao \\
\hline & Baadhi ya mazungumzo yameondolewa. \\
\hline
\end{tabular}

